\title{
A Novel Navigation Algorithm for Locomotion Interfaces with Programmable Platforms
}

\author{
Jungwon Yoon ${ }^{1}$ and Jeha Ryu ${ }^{2}$ \\ ${ }^{1}$ School of Mechanical and Aerospace Engineering and ReCAPT, \\ Gyeongsang National University, Jinju, Gyeongnam 660-701, Korea \\ jwyoonegsnu.ac.kr \\ ${ }^{2}$ Human-Machine-Computer Interface Laboratory, Department of Mechatronics, \\ Gwangju Institute of Science and Technology, Bukgu, Gwangju 500-712, Korea \\ ryu@gist.ac.kr
}

\begin{abstract}
This paper describes a novel navigation algorithm using a locomotion interface with two 6-DOF parallel robotic manipulators. The suggested novel navigation system can induce user's real walking and generate realistic visual feedback during navigation, using robotic manipulators. For realistic visual feedback, the virtual environment is designed with three components; $3 \mathrm{D}$ object modeler for buildings and terrains, scene manager and communication manager component. The walking velocity of the user is directly translated to VR actions for navigation. Finally, the functions of the RPC interface are utilized for each interaction mode. The suggested navigation system can allow a user to explore into various virtual terrains with real walking and realistic visual feedback.
\end{abstract}

\section{Introduction}

The sense of distance or orientation while walking is much better than that while riding in a vehicle for locomotion for virtual environments. Therefore, the proprioceptive feedback of walking will enhance user's immersion in most applications of virtual environments. A locomotion interface (LI) is an input-output device to simulate walking interactions with virtual environments without restricting human mobility in a confined space such as a room [1]. Fig. 1 shows the overall diagram for navigation with the LI in virtual environments, which are generated by a computer. When a human walks on the LI, the walking motions of the human are recognized by several sensors. Then, the LI utilizes the sensed information to generate infinite surfaces for continuous walking. Also, the sensed information will be transferred to virtual environments for scene update according to motions of the human walking. Finally, a human can immerse into virtual environments by feedback of visual and locomotion information.

Recently, programmable foot platforms [2-3] with robotic devices are suggested to simulate even and uneven omni-directional surfaces that are required for locomotive interactions in diverse virtual environments. Even though programmable foot platform devices can ideally simulate various terrains for natural walking, there are very few experimental reports for the programmable foot prototypes with real waking except Gait Master [2]. For allowing user's continuous walking with the limited 
workspace of the interface, Iwata [2] suggested the principal of cancellation: while one platform will follow one foot during the swing phase, the other platform will move back the other foot. However, there are no general algorithms for navigation of locomotion interface using robotic manipulators. Therefore, it is necessary to develop a generalized navigation control algorithm that allows a user to interact with virtual environments while walking over various terrains using 6-dof programmable devices. In this paper, control and navigation algorithms that can allow continuous walking over various terrains using the proposed interface are developed.

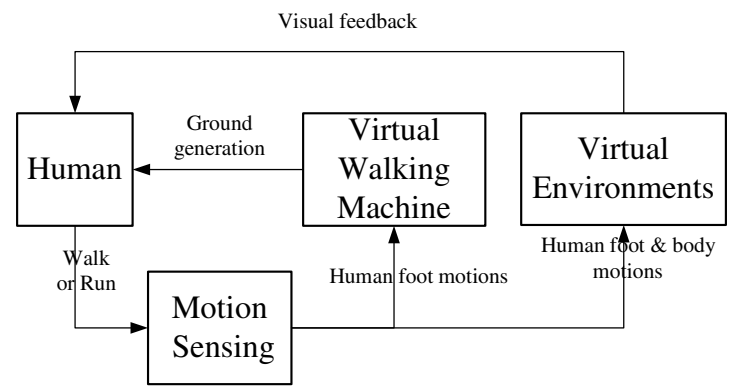

Fig. 1. Navigation using a virtual walking machine

\section{Locomotion Interface}

\subsection{Overview}

The suggested locomotion interface is composed of two planar devices on which two footpad devices are mounted. The planar device [4] is planar parallel manipulator that is composed of a platform and three limbs, each of which has three serial revolute joints (RRR) with the actuated first joint. The 3-dof footpad device [5] is composed of platform, and two limbs. The footpad device can generate pitch, roll, and heave motions at the platform.
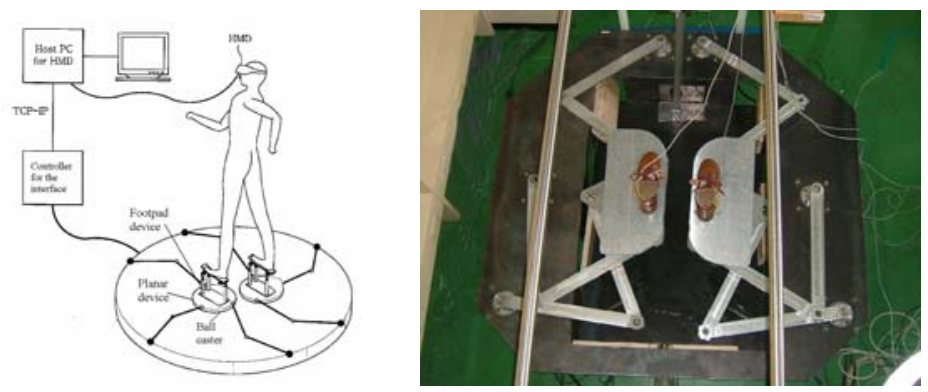

Fig. 2. The locomotion Interface with programmable foot platforms

Fig. 2 shows the structure of the proposed locomotion interface which is based on thorough understanding of the human gait. The user standing on the interface can 
walk and interact with the virtual environment while wearing a Head Mount Display (HMD), or watching big display screen. The position and orientation of a human foot can be measured using a Polhemus 3D magnetic tracker, which is tightly connected to the shoe so that it should precisely trace the foot motion without delay.

\section{Walking Control Algorithm}

The locomotion interface control system should enable a user to walk smoothly and continuously in a confined area. Thus, the control algorithm should be designed to keep the position of the human at a neutral position during walking.

\subsection{New Cancellation Method}

For a single normal gait cycle, the stance phase accounts for approximately 60 percent, while the swing phase accounts for approximately 40 percent. It should be noted however that a double support phase exists during which both limbs are in contact with the ground. During this phase, the body's center of gravity is at its lowest position. These double supports happen during initial $10 \%$ and final $10 \%$ of stance phase. Therefore, we suggest new cancellations method, in which the walking motions consider double stance phase. Thus, each platform will follow the magnetic tracker attached to a foot during swing phase when human foot is moving forward without contacting any object, while the other platform will move back during single stance phase when only one foot is in contact with ground. If two feet are in contact with the platforms, the two platforms will keep their current positions. The transitions between swing and stance phase are detected by using switch sensor system exerted by the human foot.

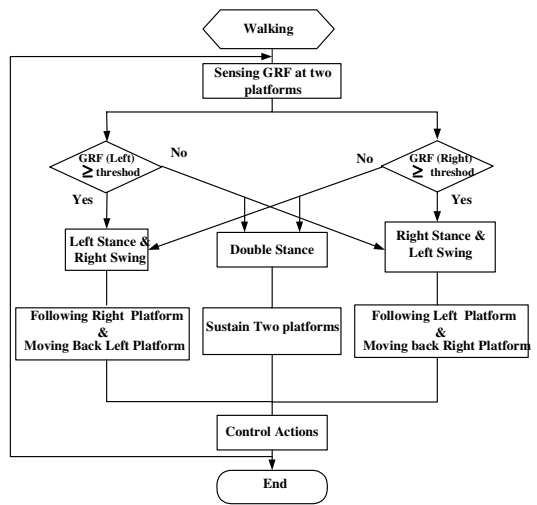

Fig. 3. New cancellation method

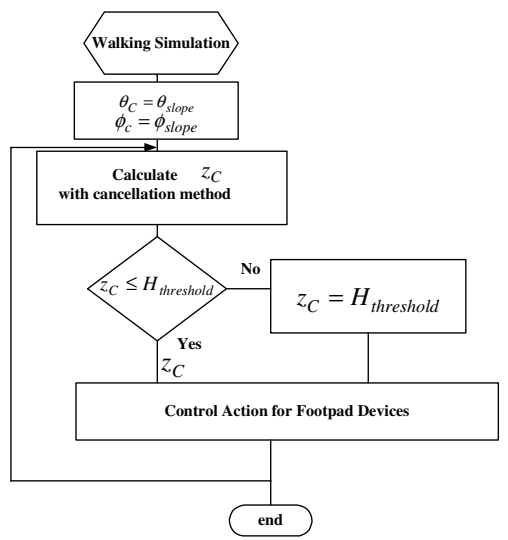

Fig. 4. Walking algorithm for spatial motions

Fig. 3 shows the block diagram of the proposed cancellation method. The proposed cancellation algorithm can allow a user to stop and start naturally according to user's intentions because of the added double support phases. Therefore, this 
algorithm will allow more natural walking on any programmable locomotion interfaces, satisfying normal gait conditions.

The proposed cancellation method is basically designed for natural walking with constant speed. However, if there is a velocity change, a human foot may be in the swing phase before or after the backward movement to the same positions with average velocities of previous swing phase during single stance phase. Due to this reason, home positioning errors generated by velocity change are inevitable. Therefore, by moving center positions of the two platforms to home positions without changing the relative positions between the two platforms, the neutral positions can be maintained although a user changes walking velocities.

\subsection{Spatial Motions}

For spatial motions of the locomotion interface, the footpad device will be used to generate various terrains such as stairs and slopes. In order to simulate stairs, the platform of the footpad device should have zero angles since the stairs have no slope. Therefore, cancellation method about planar motions can also be applied to lift motion control. If the $z_{C}$ is higher than the ground height $H_{\text {threshold }}$, the command lift motion $z_{C}$ of the footpad device will be $H_{\text {threshold }}$ to keep stairs surface. This algorithm for spatial motions is shown in Fig. 4. For slope surface generation, if the pitch angles of the footpad device have constant values and the roll angle is zero, the surface will be uphill or downhill slopes. If ground has up-slope, the pitch angle should be positive and the ground height should be increased as human foot proceeds in forward direction as shown in Fig. 5. Therefore, to sustain the contact of the human foot with the ground at slope surfaces, the ground height threshold $H_{\text {thereshold }}$ should be computed in equation (1), while the same walking scheme for planar motions and lift motions will be applied to retreat the human foot back for continuous walking.

$$
\theta_{C}=\theta_{\text {slope }}, \phi_{C}=\phi_{\text {slope }}, H_{\text {threshold }}=H_{\text {ground }}+y_{C} \tan (\theta)
$$

where $\theta_{C}$ and $\phi_{C}$ are the desired pitch and roll angles of the footpad device, and $y_{C}$ is the back-and-forth desired control command of a human foot. This walking control algorithm, therefore, will sustain continuous walking over various terrains with the 6-dof locomotion interface in a limited area.

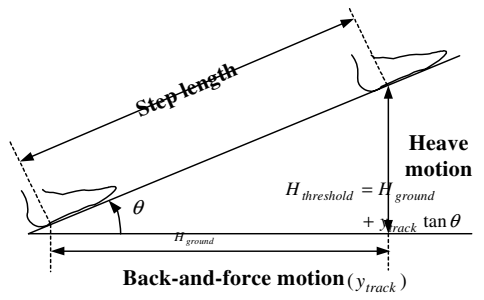

Fig. 5. The walking surface generation on slopes 


\section{Navigation Using the Locomotion Interface}

\subsection{Virtual Environments}

Virtual terrains (see Fig. 6) have been developed by using OpenGL API (Application Program Interface) based on Microsoft Visual $\mathrm{C}++$ programs for window environment. The viewpoint is selected as the first person viewpoint so that operator may see virtual environment and feel more realistic. Virtual environments are displayed with large screen to guarantee safety of the subject during the locomotion interactions. Even though HMD (Head Mounted Display) can provide full sight of virtual environment, the wearing of the HMD may prevent the user from coping with dangerous situations during walking interactions on the locomotion interface.

The virtual environment consists of three components:3D object modeler for buildings and terrains, scene manager and communication manager component. The 3D object modeler component provides geometric modeling functionality for virtual environment through polygon extrusion of $2 \mathrm{D}$ profile in the $2 \mathrm{D}$ map and $3 \mathrm{D}$ CAD model. The 3D model can be created for 2D map by extruding 2D profile geometry with $b$ and $m$. In simple features specification of the Open GIS Consortium (OGC)[6] , the WellKnown Binary (WKB) representation for geometry provides a portable representation of a geometry value as a contiguous stream of bytes. Also, WKB representation is used to store geometry information as in OGC simple features specification.

The scene manager component manages the scene graph for real-time rendering according to the VWM requests. The communication manager component manages the communication between virtual walking machine and virtual environment via remote procedure call (RPC) to interact the VWM user. The interface using the interface definition language (IDL) for RPC is defined to communicate between the VWM and virtual environment. The proposed RPC interface provides virtual environment switching and interaction functions.

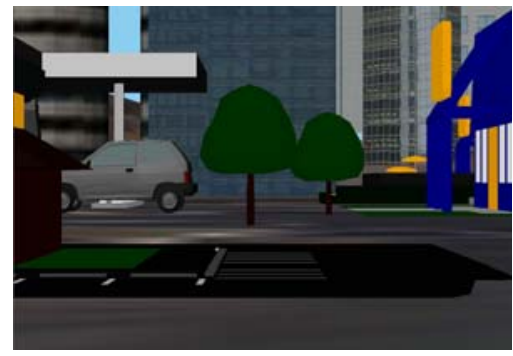

(a) Upright navigation

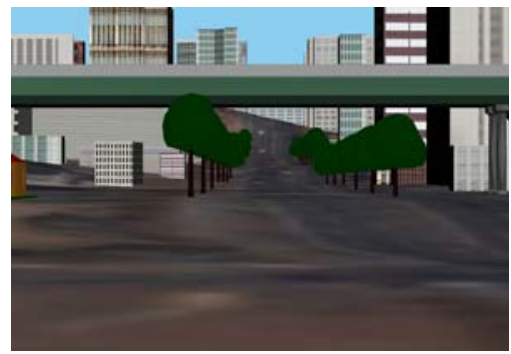

(b) Slope navigation

Fig. 6. Virtual terrains

\subsection{Interaction Between the LI and Virtual Environments}

There are two types of RPC methods for interaction: position-based interaction and action-based interaction. The position-based interaction performs the navigation in virtual environments according to the position changes in local coordinate system of the VWM, while the action-based interaction performs relative motion at current virtual environments according to the velocity changes of the VWM. Since there are 
many virtual environments with various terrains, the action-based interaction is easy to control the virtual environments in that it performs the navigation according to the VWM user actions rather than the position changes. Therefore, the walking velocity of the user will be directly translated to VR actions for navigation. The update rate for the VWM velocity is selected to be $20 \mathrm{~Hz}$, which is enough to show smooth walking actions. Then, the user walking velocity is estimated as;

Single Right Stance Phase:

$$
V_{\text {walking }}=V_{\text {avg }, L}^{s w}
$$

Single Left Stance Phase:

$$
V_{\text {walking }}=V_{\text {avg }, R}^{s w}
$$

Double Stance Phase:

$$
V_{\text {walking }}=0
$$

where $V_{a v g, i}^{s w}$ is the average velocity during the swing phase and the $V_{\text {walking }}$ is the estimated walking velocity of a user on the locomotion interface. Since human foot moves forward only during swing phase, the average velocity of the human walking can be estimated as average velocity during only swing phase motion. Therefore, after the each foot moves forward and when the foot contacts on the platform, the walking velocity will be updated. During double limb stance, since the foot will not move forward, the velocity should be set to zero. Finally, the functions of the RPC interface shown in Fig.7 are utilized for each interaction mode.

The functions Forward (speed) and Backward (speed) for back-and forth motions will move the current camera position to the next camera position towards the direction vector of the current camera. The speed will determine differential displacement during sampling time. Similarly, the functions UpStair(speed) and DownStair(speed) will change the camera position of virtual environments for $\mathrm{z}$ direction. The functions LookLeft (angle), LookRight (angle), LookUp (angle), and LookDown (angle) are defined to change directions of the camera, which will be connected to HMD(Head Mounted Display) to reflect the change in user's view in virtual environments.

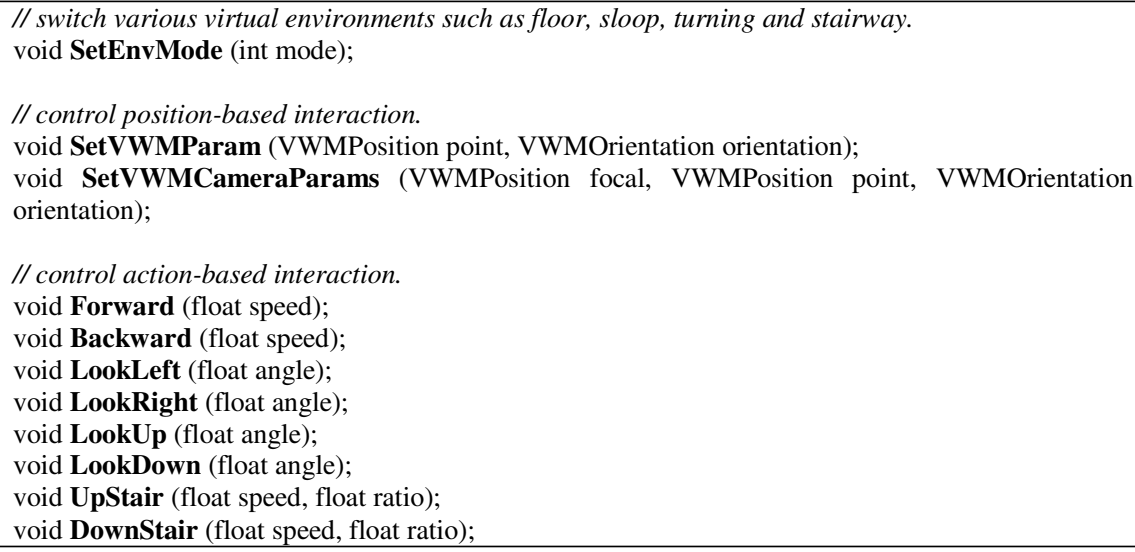

Fig. 7. RPC interface for interaction between the VWM and virtual environments 


\subsection{Evaluations}

For the preliminary interface evaluation, five subjects among our laboratory students participated in walking over the designed locomotion interface device. The subjects who have no experience of the walking on the interface were instructed how to walk over the walking interface. Since there is velocity limitation (maximum $1.2 \mathrm{~m} / \mathrm{s}$ ) for the locomotion interface, they were requested to walk with normal speeds that they generally walk. Even though the number of subjects is small, it is useful for discussing the overall performance of the walking interface. Initially, most of them were afraid of the waking on the interface but after trial walking, they were able to walk without anxiety. Fig. 8 (a) shows the scores of the each item (safety, reality, and overall) for upright walking with respect to subjects A...E. After interactions with the walking device, they scored each item. Most of them are satisfied with the walking control algorithm since the algorithm has been developed based on the real human gait motions. But, they indicated that for moving back motions during stance phase, they felt the body inertia of moving back motions due to the neutral home positioning during double stance phase. For overall evaluations, subjects generally were satisfied with the reality of the walking with the interface device. For slope walking on the interface shown in Fig. 8 (b), the subjects were more positive for real walking in that there was not much of the body inertia for backward motions, especially for the backand-forth motions. Since real bodies were moving up and moving down for walking interaction on slope grounds, they felt that their walking motions were more similar to real walking. For turning motions [7-8], since the two platforms may have possibility to collide each other when the platform follows the human foot, the only passive motions are allowed.

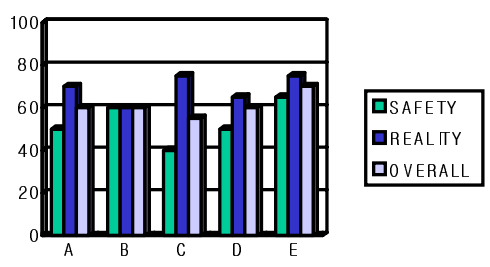

(a) upright walking

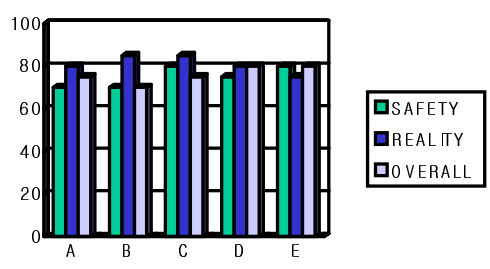

(b) slope walking

Fig. 8. Evaluation results

With preliminary evaluations, some important understandings of the developed locomotion interface are addressed. The walking control and navigation algorithm was operating well with the proposed novel programmable locomotion interface. The walking interactions with the upright and slope grounds were successful on the locomotion interface. 


\section{Conclusions $^{1}$ and Future Work}

This paper proposes novel navigation system that can induce user's real walking and generate realistic visual feedback during navigation, using robotic manipulators. For realistic visual feedback, the virtual environment is designed with three components; 3D object modeler for buildings and terrains, scene manager and communication manager component. The suggested navigation system can allow a user to explore into various virtual terrains with real walking and realistic visual feedback. As future works, the various haptic effects such as soft and hard grounds, or slippage will be simulated by changing the impedance parameters. Also, comparison between VWM and joystick navigation will be performed. After enhancing the control performances and the safety of the VWM, the final goal is to let user wear the HMD for full immersion in the virtual navigation with natural walking.

\section{References}

1. Hollerbach, M.,"Locomotion interfaces," in: Handbook of Virtual Environments Technology, K.M. Stanney, ed., Lawrence Erlbaum Associates, Inc. (2002) 239-254.

2. Iwata, H., Yano, H., and Nakaizumi, F., Gait Master: a versatile locomotion interface for uneven virtual terrain, Prof. of IEEE Virtual Reality (2001) 131 - 137.

3. Schmidt, H., Sorowka, D., Hesse, R., and Bernhardt, R., Design of a Robotic Walking Simulator for Neurological Rehabilitation, IEEE/RSJ Int. Conf. On Intelligent Robots and Systems (2002) 1487-1492.

4. Yoon, J. and Ryu, J., The Development of the 3-DOF Planar Parallel Robot (RRR Type) for Omni-directional Locomotion Interface, $3^{\text {rd }}$ IFAC Symposium on Mechatronic Systems, Sept.6-8, Sydney, Australia, 2004.

5. Yoon, J. and Ryu, J., A New Family of 4- Dof Hybrid Parallel Mechanisms with Two Platforms and Its Application to a Footpad Device, Journal of Robotic Systems, 22:(5) (2005) 287-298.

6. Open GIS Consortium, OpenGIS Simple Feature Specification for OLE/COM, OpenGIS Implementation Specifications, Revision 1.1, 1999.

7. Bouguila, L., Ishii, M., and Sato, M., Realizing a New Step-in-place Locomotion Interface for Virtual Environment with Large Display System. Proceedings of the Workshop on Virtual Environments (2002) 197-207.

8. http://www.virtusphere.com.

1 Research reported here was supported by grant (No. R01-2002-000-00254-0) from the Basic Research Program of the Korea Science \& Engineering Foundation and by the Korea Research Foundation Grant funded by the Korean Government(MOEHRD)" (KRF-2005-005-J09902). 